Revue de recherche interdisciplinaire sur le genre et la sexualité

35 | 2018

Striges en tous genres

\title{
De la liminalité de la grosseur : stratégies spectaculaires et identité de gros
}

Camille Ronti

\section{OpenEdition}

\section{Journals}

Édition électronique

URL : https://journals.openedition.org/sextant/387

DOI : $10.4000 /$ sextant.387

ISSN : 2795-8736

\section{Éditeur}

Éditions de l'Université de Bruxelles

\section{Édition imprimée}

Date de publication : 1 décembre 2018

Pagination : 87-99

ISBN : 978-2-8004-1636-6

ISSN : 1370-267X

\section{Référence électronique}

Camille Ronti, « De la liminalité de la grosseur : stratégies spectaculaires et identité de gros », Sextant [En ligne], 35 | 2018, mis en ligne le 01 novembre 2021, consulté le 08 décembre 2021. URL : http:// journals.openedition.org/sextant/387 ; DOI : https://doi.org/10.4000/sextant.387

\section{c) (i) (2)}

La revue Sextant est mise à disposition selon les termes de la Licence Creative Commons Attribution Pas d'Utilisation Commerciale - Partage dans les Mêmes Conditions 4.0 International. 


\title{
De la liminalité de la grosseur : stratégies spectaculaires et identité de gros
}

\author{
Camille RonTi
}

\section{Introduction}

Cet article vise à exposer certaines stratégies spectaculaires et identitaires pour comprendre le corps, non comme une entité ontologique vierge, mais bien comme un construit social, traversé par des relations de pouvoir qui le créent. Plus encore, il se concentre sur le corps gros, à travers deux cas d'étude clés : d'une part, le groupe militant Fat Positivity Belgium, actif dans le milieu queer bruxellois et, d'autre part, la troupe de danse contemporaine australienne Force Majeure et son spectacle Nothing to Lose. En dévoilant la fausse naturalité du corps normé (à savoir, mince) et donc non stigmatisé, l'intérêt est de prôner une diversité corporelle, déstabilisant les dynamiques de pouvoir induites par le discours normalisateur, et ainsi de comprendre le corps gros comme un corps historiquement et socialement pathologisé, marginalisé, rejeté dans l'anormal. Voilà tout le travail des artistes et militants étudiés dans la présente analyse. Nous examinerons la manière dont ces pratiques performatives permettent de dévoiler les rapports de force sous-tendus par l'hégémonie de la minceur dans la société occidentale néo-libérale contemporaine, ainsi que la subversion en termes d'identité que ces pratiques engendrent.

Le concept central dans cette recherche est celui de performance. Recouvrant des réalités différentes, bien qu'étroitement liées, la performance est un terme défini par l'indéfinissable, à l'instar de l'identité. La performance permet de dévoiler la contingence des structures de pouvoir sous-jacentes à la société et de les redéfinir. La performance a donc un haut potentiel subversif en ce qu'elle permet de réinventer des modèles de pouvoir existants. Notons que le corps sera toujours ici envisagé comme une performance culturelle, c'est-à-dire une performance ancrée dans un contexte culturel particulier (ici, la société occidentale contemporaine - patriarcale, néo-libérale). Il s'agira donc du corps gros, sujet aux injonctions à la minceur, qui 
maintiennent les corps dans une binarité stricte (mince/gros, blanc/de couleur, homme/ femme, etc.) et discriminent les personnes dont les corps ne correspondent pas à la norme. La notion de performance culturelle est à rapprocher de celle de «pratique performative ». Celle-ci se définit, selon Pradier, comme une pratique qui met au centre de son attention le corps, véritable « fil conducteur » posant problème puisqu'il relève de conceptions différentes selon les " horizons culturels " ${ }^{1}$ et qui ne doit se comprendre qu'en relation avec ceux-ci - c'est ainsi que la grosseur ne doit être envisagée qu'à travers le prisme de la société occidentale contemporaine, ici, qui la médicalise et la rejette dans l'a-normal.

Ce que les artistes et militants gros réfutent, c'est la liminalité de leur identité de gros. Il s'agit, comme l'explique la chercheure Harjunen, de cet état temporaire dans lequel est obligatoirement placé le corps gros ${ }^{2}$. L'individu gros ne peut le rester et doit se soumettre à des processus d'amincissement (régime, sport, chirurgie) pour retrouver son " état originel de mince », son état normal. Cette liminalité ne permet ainsi pas la sujétion de l'individu. En outre, elle induit et sous-tend des rapports de force entre les individus qui maintiennent l'individu dans des binarités strictes. Un outil clé dans ces dynamiques de pouvoir est le discours prononcé par des institutions de pouvoir (ici, du corps médical). Le discours, selon Foucault, est une pratique, ancrée dans un contexte historique, qui produit du pouvoir et du savoir, et qui soustend des institutions et des groupes sociaux ainsi que leurs relations ${ }^{3}$. Nous verrons ainsi les discours qui légitiment la norme de minceur.

Depuis plusieurs décennies, dans la société occidentale néo-libérale, l'idéal de beauté et de santé est la minceur. Devenue véritable dictature corporelle, la minceur représente une hégémonie normative. «Dominance souveraine exercée sur quelque chose $»^{4}$, concept repris par Gramsci qui parlait, lui, notamment d'« hégémonie culturelle », ce terme désigne parfaitement la relation de domination/soumission entre différentes classes et catégories sociales. Il s'agit donc de la catégorie dominante, la norme, et du pouvoir normalisateur qu'elle exerce sur les corps qui n'y sont pas conformes - le corps mince normé et normal versus le corps gros. Tout un éventail de présupposés compose et renforce cette hégémonie. L'hégémonie de la minceur repose sur l'imaginaire collectif qui entoure la grosseur : image de la surconsommation, synonyme de surabondance alimentaire, incapacité à se contrôler, à être maître de soi, égoïsme, corps sans gêne, etc. En somme, la grosseur est devenue, depuis plusieurs années maintenant, le Mal du Siècle.

\section{Le militantisme gros, késako ?}

Pour contrer ces conceptions hégémoniques du corps gros, un mouvement est né aux Etats-Unis, à la fin des années 1960. Comme l'explique Charlotte Cooper, militante

\footnotetext{
1 J. DuBoIs, « Le corps comme étalon de mesure », M@gm@, 7/3, septembre-décembre 2009.

2 H. HarJunen, « Obesity as a Liminal and Marginalized Experience », présenté à la $5^{\mathrm{e}}$ conférence de recherche féministe européenne, à l'Université de Lund, Suède, en 2003.

3 T. Spargo, Foucault and queer theory, Cambridge, Icon books, 1999.

${ }^{4}$ http://www.tresor-de-la-langue-francaise informatise.fr/dendien/scripts/tlfiv5/advanced. exe?8;s=2389780440.
} 
grosse britannique, la première organisation a été la NAAFA (National Association to Advance Fat Acceptance), créée en 1969 à l'initiative de William Fabrey afin de prendre part au mouvement pour les droits civiques (à l'instar du mouvement de libération des femmes, des gays et du Black Power). Ainsi, cette première vague de militantisme émerge d'un mouvement grass roots et est profondément ancrée dans un combat plus large : l'abolition de toutes les discriminations. Dès le milieu des années 1970, des dissonances se font entendre au sein du groupe : Aldebaran et Freespirit, toutes deux partisanes de la Radical Feminist Theory, décident de fonder le Fat Underground. Ces deux mouvements fondateurs se positionnent comme anti-essentialistes ${ }^{5}$, notion cruciale dans ce travail de déconstruction des normes. Le mouvement, d'origine américaine, a fini par avoir une contrepartie en Europe. Le Royaume-Uni a suivi, dans les années 1980, grâce notamment au Fat Women's Group, au magazine Extra Special ou encore au livre de Shelley Bovey : Being fat is not a Sin.

Notons que ce «mouvement » ne dépend pas d'un fondement théorique ou organisationnel à la base et consiste souvent en des personnes grosses qui militent de manière plutôt isolée ${ }^{6}$. Par conséquent, il existe à l'heure actuelle maintes branches $\mathrm{du}$ « mouvement », avec toutefois une idée commune libérationniste ${ }^{7}$ : lutter contre la discrimination envers les personnes grosses, promouvoir la diversité corporelle, rejeter l'oppression systémique des corps liée à un idéal de beauté. Le corps devient objet et sujet de revendication politique, il devient le moyen d'expression d'une revendication sociale à disposer de son corps personnel. Soulignons, par ailleurs, la perspective de genre dans laquelle s'inscrit le mouvement : les discriminations liées à la grosseur ne sont pas identiques pour les femmes et pour les hommes. En effet, les femmes grosses sont parfois victimes de violence justement à cause de leur corps ${ }^{8}$ et le seuil d'acceptation sociale varie pour les femmes et pour les hommes ${ }^{9}$.

S'il fallait résumer la vocation des militants gros, il suffirait de lire une citation d'Allyson Mitchell : « Fat activism is about doing something. It is about changing things. What do we want to change ? Just about everything you can think of when

${ }^{5} \mathrm{~K}$. LeBESCO, Revolting bodies ? The struggle to redefine fat identity, Cambridge, University of Massachusetts Press, 2004, p. 14. La position essentialiste se caractérise par l'idée que l'état de grosseur est nécessaire et dû à un antécédent ou à un choc traumatique. Ainsi, l'essentialisme prône que l'identité de gros est un résultat inévitable, qui a toujours pour cause un évènement qui a mal tourné. Comme l'explique LeBesco, la position anti-essentialiste consiste à repositionner l'être humain au centre de la production de signification, par des processus discursifs et politiques.

${ }^{6}$ Ch. CoOper, Fat and proud: The politics of size, Women's Press (UK), 1998, p. 146.

${ }^{7}$ K. LeBesco, Revolting bodies ?, op. cit., p. 42. LeBesco définit le militant assimilationniste comme celui qui considère la grosseur comme un problème, à l'inverse du libérationniste qui, lui, la célèbre.

${ }^{8}$ T. Royce, "The Shape of Abuse », in E. Rothblum et S. Solovay, The fat studies reader, New York, New York University Press, 2009, p. 151.

${ }^{9}$ H. HARJUnEN, Women and fat : Approaches to the social study of fatness, University of Jyväskylä, 2009, p. 27-28. 
it comes to how fat and fat people are treated, thought about, and represented in our society $\gg{ }^{10}$.

Ainsi donc, depuis la fin des années 1960, des personnes grosses, militantes et/ou artistes, tentent de déconstruire l'hégémonie de la minceur, de dévoiler les rapports de forces qui sous-tendent cette dictature de la santé et de proposer une représentation positive de la grosseur. Alors, quelles sont les pratiques subversives en vigueur dans le plat pays?

En Belgique, le groupe de militantisme gros, Fat Positivity Belgium ${ }^{11}$, a vu le jour en janvier 2013. Au carrefour entre féminisme et militantisme queer ${ }^{12}$, le collectif propose des ateliers, des groupes de parole et des séminaires de lectures autour du sujet, et travaille sur la base du corps gros, pouvant être lu comme une performance culturelle qui dévoile les structures de forces sous-jacentes et permet de les réinventer. Par ailleurs, il est à la base de plusieurs actions militantes ${ }^{13}$ promouvant la visibilité des personnes grosses et collabore avec d'autres associations, actives dans le milieu queer.

Le message du groupe ? «Fat Positivity est un groupe belge d'activisme gros. Dans une perspective féministe, queer et intersectionnelle, nous luttons pour un monde dans lequel tous les corps sont de bons corps, qui méritent le respect et sont respectés ! Nous vous convions à nos performances, nos groupes de lecture et de discussions (consciousness-raising), nos échanges de vêtements et autres activités ludiques en français, néerlandais et anglais. La haine ne passera pas par nous ! : Fat Positivity Belgium rejette tous discours de haine, en ce compris, le fat-shaming, le thin-shaming, le racisme, la transphobie, l'homophobie, le sexisme, l'âgisme, le validisme, etc. ».

\section{Du discours et des rapports de force}

Fat Positivity met un point d'honneur à déconstruire les discours hégémoniques de la grosseur. En effet, du point de vue de la discipline des corps, il est nécessaire de parler du discours, qui véhicule et produit du pouvoir ${ }^{14}$, car « tout rapport de pouvoir s'exerce sur, à travers et au moyen des corps ${ }^{15}$, moyen par lequel le discours se voit, littéralement, « incorporé » à l'individu. Pour Foucault, le pouvoir doit se comprendre comme une multiplicité de rapports de force, fluides et émanant de partout, c'est-à-dire de toutes les strates des institutions disciplinaires constituant

10 D. Kulick et A. Meneley (éd.), Fat : The anthropology of an obsession, New York, Jeremy P. Tarcher/Penguin, 2005, p. 212.

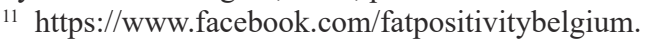

12 http://www.TransQueerWellness.org, J. Skelton, Oberlin College, 2007, et B. G. BeEmyn, GLBT Student Services, Ohio State University, 2006, Queer Glossary. La définition du terme queer dans ce glossaire est : " terme reprenant une vaste matrice d'identités extérieures à la majorité monogame, hétérosexuelle et de genre normé ».

${ }_{13}$ A savoir, des groupes de parole, des tables de discussions consciousness-raising, des collaborations avec des festivals queer bruxellois, des soirées à thème, des performances artistiques, des ateliers et du cyber-militantisme.

${ }_{14}$ M. Foucault, Histoire de la sexualité, I, La volonté de savoir, Paris, Gallimard, 1976, p. 133.

15 A. SforzInI, Michel Foucault : une pensée du corps, Paris, PUF, 2014, p. 39. 
la société et non pas d'une instance souveraine ${ }^{16}$. En outre, le discours produit et véhicule du pouvoir mais dissimule aussi les rapports de force ${ }^{17}$. En résumé, la normalisation (c'est-à-dire l'instauration de la norme) est un effet de ce pouvoir et ce dernier est « normalisé » parce que dissimulé au sein des discours hégémoniques ${ }^{18}$. Ainsi, le corps, tant individuel que social, est traversé par un ensemble de discours producteur de pouvoir et se voit par conséquent discipliné, rendu docile, normalisé. Ce pouvoir est également savoir et produit donc une connaissance sur le corps, dont la fausse naturalité engendre l'hégémonie, la toute-puissance. A défaut de parvenir à correspondre aux attentes normalisatrices, l'individu dont le corps serait hors-normes se voit marginalisé et son identité, menacée. Les personnes grosses, dont le corps ne correspond pas aux normes de minceur en vigueur véhiculées par bon nombre d'instances de pouvoir (politique, médicale, médiatique...), ne peuvent accéder à une identité sociale viable : les discours hégémoniques leur rappellent la nécessité de se conformer ou de payer le prix.

Pour comprendre l'essence même du militantisme gros, il est capital d'envisager la grosseur dans un système de discriminations plus vaste. Ce système, appelons-le capitalisme, patriarcat, ou autre, repose sur l'institutionnalisation des rapports de force par le biais du discours. Ce discours normalisateur s'immisce au plus profond du corps et marque les corps anormaux au fer rouge. Mais si le discours institutionnalisé a du pouvoir, il n'est pas le seul. C'est bien là tout l'intérêt des discours alternatifs des mouvements militants et toute l'importance de se réapproprier une terminologie. C'est la raison pour laquelle le terme gros est utilisé ici et que les termes tels que « en surpoids », « rond.e » ou « en chair » sont évacués du vocabulaire des militants.

Par ailleurs, il est nécessaire de s'accorder sur le terme « grossophobie » ${ }^{19}$, composé du préfixe « gros » et du suffixe « phobie » (à l'instar de l'homophobie, par exemple), qui sera utilisé pour parler des préjugés, discriminations ou stigmatisations associés à la grosseur. L'auteure et professeure Kathleen LeBesco utilise le terme « révoltant » pour caractériser l'image que façonnent les préjugés dont font l'objet les personnes grosses ${ }^{20}$. Ainsi, pour elle, ce terme englobe la perception du corps gros comme « malsain », ou encore « déplaisant», mais aussi la représentation de la grosseur comme agent de « dégoût et d'aversion ». Par ailleurs, plusieurs adjectifs sont associés au corps gros dans le discours hégémonique et il est ainsi perçu comme 《 impropre ${ }^{21}$ ou encore «paresseux ${ }^{22}$. Il est déjà possible de noter différents aspects de ce phénomène : d'une part, la grosseur semble être liée à la mauvaise santé (et ce préjugé tend à une pathologisation de la grosseur, l'associant nécessairement

${ }^{16}$ M. Foucault, Histoire de la sexualité, op. cit., p. 133.

17 J. BUtLER, Le pouvoir des mots : discours de haine et politique du performatif, Paris, Editions Amsterdam, 2004, p. 80.

${ }^{18}$ H. HaRJunen, Women and fat, op. cit., p. 33.

19 Traduction, admise par les militants gros francophones, de l'anglais fatphobia.

${ }^{20}$ K. LeBesco, Revolting bodies ?, op. cit., p. 1.

${ }^{21}$ J. E. Braziel et K. LeBesco (éd.), Bodies out of bounds : Fatness and transgression, Berkeley, Los Angeles, London, University of California Press, 2001, p. 1.

${ }^{22}$ D. Schuster et L. Tealer, " Exorcising the Exercise Myth », in E. Rothblum et S. SolovaY, The fat studies reader, New York, New York University Press, 2009, p. 320. 
à un état pathologique, malade) ; d'autre part, elle va à l'encontre d'un idéal de beauté, d'une esthétique normative. Comme l'explique Pierre Fraser, il est possible de distinguer deux formes de stigmatisations liées à la grosseur : celle basée sur le jugement moral d'autrui et celle engendrée par les interventions publiques ${ }^{23}$. Par conséquent, la personne grosse se voit attaquée sur deux fronts : le corps personnel (jugé en tant qu'individu par d'autres individus) et le corps social (jugé en tant que partie problématique de la population, par les interventions publiques qui visent à l'effacer de l'espace public). Outre le jugement moral porté sur le corps individuel, les personnes grosses doivent faire face à des discriminations quotidiennes d'ordre public. C'est ainsi que Cooper aborde l'environnement grossophobe ambiant (notamment concernant l'infrastructure des transports publics) ${ }^{24}$, mais aussi la discrimination dans le milieu du travail (une personne grosse aurait ainsi moins de chance de se faire engager ou de recevoir une promotion) ${ }^{25}$. D'après Paul Ernsberger ${ }^{26}$, la grosseur engendrerait un risque d'être au chômage plus élevé ou d'avoir un revenu plus faible, et cela à cause du climat grossophobe actuel. Il analyse ensuite une étude effectuée (par un chercheur de l'université d'Harvard sur la pauvreté et les populations minoritaires ainsi que sur la santé des enfants et adolescents) auprès d'adolescents minces et gros et sur leurs conditions de vie sept ans plus tard. Chez les femmes, l'écart se fait beaucoup plus sentir que chez les hommes. Selon l'étude, seules $27 \%$ des filles grosses étaient mariées (contre $56 \%$ des filles minces), leur revenu était un tiers moins élevé et elles avaient trois fois plus de risque de vivre sous le seuil de pauvreté.

Soulignons-le, comme l'indique Hartley, la grossophobie est un des rares préjugés encore acceptables à l'heure actuelle ${ }^{27}$. Ainsi, les différentes formes que prennent les discriminations envers les personnes grosses ne semblent pas reconnues comme portant atteinte à l'individu et restent politiquement correctes : la grossophobie est toujours acceptée et se voit même, dans certains cas, encouragée.

\section{Liminalité, ce cercle vicieux à briser}

Quelles sont les implications de la grosseur en ce qui concerne l'identité sociale et individuelle ? C'est tout le travail d'un groupe militant, qui utilise le personnel pour lutter contre le politique, le systémique.

La chercheure finlandaise Harjunen s'est intéressée à la question du corps gros de la femme et c'est ainsi que, selon elle, le corps gros de la femme se trouve « à l'intersection entre différents discours politiques définissant les limites de l'acceptable et de l'inacceptable ${ }^{28}$. Le corps gros se retrouve par conséquent exclu, hors-normes. C'est dans cette perspective d'inacceptabilité du corps gros qu'Harjunen a postulé

${ }^{23}$ P. FRASER, Le corps obèse objet d'aversion. 1. De la Renaissance jusqu'au XIXe siècle, Editions vf/Tel-T-Textes, 2014, p. 89.

${ }^{24}$ Ch. COOPER, Fat and proud, op. cit., p. 19.

${ }^{25}$ Ibid., p. 23.

${ }^{26}$ P. ErnSBerger, « Does Social Class Explain the Connection Between Weight and Health ? », in E. Rothblum et S. Solovay, The Fat Studies Reader, New York, New York University Press, p. 26-27.

${ }^{27}$ C. Hartley « Letting Ourselves Go », in J. E. Braziel et K. LeBesco, op. cit., p. 65.

${ }^{28}$ H. Harjunen, Women and fat, op. cit., p. 19. 
l'idée de liminalité de la grosseur ; dans Women and $\mathrm{fat}^{29}$, elle explique que, selon ses recherches, la grosseur est considérée surtout comme un état transitoire, non permanent, donc liminal. Cet état temporaire est sous-tendu par le discours médical, qui insiste sur la nécessité de guérir la grosseur. Cette liminalité maintient les corps gros dans un état de transition, qu'il n'est pas possible d'assumer de manière permanente, qu'il faut soigner, pour se conformer à la norme et devenir ainsi viable. Ainsi, un paradoxe apparaît : l'individu ne peut s'identifier à une catégorie sociale de « gros », ne peut se construire en tant que sujet « gros », doit sans cesse être à la recherche de son identité « mince », seule identité valable, et par conséquent renforce l'idée de liminalité de la catégorie " gros ». Par conséquent, être gros n’est pas une identité, il n'existe qu'un sujet « mince », temporairement gros, qui cherche à se conformer à la norme, afin de devenir sujet à part entière. Ainsi, chaque tentative de normalisation (régime, sport, chirurgie, etc.) semble témoigner de la volonté de l'individu de devenir viable, de rejeter son statut liminal et de se conformer à une identité sociale vivable. Ces tentatives se voient encouragées, valorisées ; elles deviennent le symbole d'une « auto-libération », d'une capacité individuelle de modifier son corps à son gré, d'être maître de soi ${ }^{30}$. Toutefois, selon les recherches d'Harjunen, il apparaît aussi que cet état de grosseur liminale devient en définitive permanent, car les outils mis en place ne sont pas efficaces et ainsi la liminalité obligatoire des personnes grosses devient définitive.

Certaines stratégies militantes visent directement cette liminalité : se réapproprier le terme " gros.se ", proposer une représentation positive des personnes grosses loin des stigmates de l'hégémonie de la minceur ou encore revendique clairement la disparition de l'identité liminale de gros et l'accès à une identité sociale viable et positive.

\section{L'intersectionnalité, concept clé}

Pour comprendre le combat des militants gros, il faut aussi l'envisager dans une perspective intersectionnelle. Ce principe doit se comprendre comme un outil d'étude des rapports de force qu'engendre la norme et donc d'étude de discriminations (liées à l'identité sociale associée à l'ethnie, au genre, à la sexualité, à la religion, à la classe, à l'âge, à la grosseur ou encore au handicap) comme mécanismes de pouvoir qui coproduisent exclusion et marginalisation ${ }^{31}$. Ces constructions sociales s'entremêlent et ne peuvent être envisagées séparément car elles dépendent de dynamiques de pouvoir et d'oppression similaires et liées, qui se sous-tendent, se renforcent l'une l'autre. Ces catégories reposent sur une conception binaire de l'identité de l'individu (par exemple, homme/femme, blanc/de couleur, valide/invalide, mince/gros...) et sous-tendent une dynamique de privilèges accordés à la catégorie dominante, au détriment de la catégogie subordonnée ${ }^{32}$. Ainsi, la discrimination, c'est-à-dire les

\footnotetext{
${ }^{29}$ Ibid., p. 62-64.

${ }^{30}$ L. Gilman Sander, Fat : A cultural history of obesity, Hoboken, John Wiley \& Sons, 2013 , p. 6.

${ }^{31}$ N. Van Amsterdam, «Big fat inequalities, thin privilege : An intersectional perspective on « body size » », European Journal of Women's Studies, 2013 p. 155-156.

32 H. HarJunen, Women and fat, op. cit., p. 58.
} 
différents désavantages et stigmatisations liés à une catégorie, une identité sociale non privilégiée, est le revers d'une dynamique de pouvoir et l'intersectionnalité vise à reconnaître que les discriminations opèrent sur plusieurs fronts à la fois, que des liens sont à établir entre les différentes identités sociales construites ${ }^{33}$. Une discrimination liée à une identité sociale en cache souvent une autre liée à une autre identité sociale et tend à la renforcer.

Ainsi, il est capital de comprendre que le lieu privilégié de cette intersection est le corps ${ }^{34}$ et qu'à ce titre, tout corps perçu comme anormal sera le lieu de combats et de discriminations dans une société de productivité, de compétitivité et de rentabilité à tout prix. La lutte des militants gros doit par conséquent se lire dans une perspective de genre, post-coloniale et queer.

Les actions menées par Fat Positivity Belgium ${ }^{35}$ ont été multiples : groupes de parole, projection de films militants dans le cadre du festival Pink Screens ${ }^{36}$, performance artistique, échanges de vêtements, rencontre avec des militantes grosses reconnues telles que Charlotte Cooper... En somme, les activités et les actions du groupe se sont diversifiées et ont reflété l'essence même de son message : être queer, être intersectionnel, être présent et affirmer sa grosseur pour atteindre une identité socialement viable. Une multiplicité d'actions, pour un combat sur plusieurs fronts à la fois. Les actions, pour la plupart menées au sein de la RainbowHouse, ont réaffirmé l'intersectionnalité du groupe.

\section{La médicalisation de la grosseur, une avancée des Lumières}

Un des combats clés pour le groupe Fat Positivity Belgium est la médicalisation de la grosseur car il s'agit de la base du modèle hégémonique et de la source de légitimité de la grossophobie actuelle. Les préjugés et discriminations dont sont victimes les personnes grosses ont été engendrés par la médicalisation de la grosseur, à la base du « modèle dominant ». Aujourd'hui, le « surpoids » et l'« obésité » sont définis par l'Organisation mondiale de la Santé comme « une accumulation anormale ou excessive de graisse corporelle qui représente un risque pour la santé ${ }^{37}$. Toutefois, le corps gros n'a pas toujours été dans la ligne de mire du discours médical ; c'est ce dont atteste, en effet, l'apparition du terme « obésité » dans le dictionnaire d'Antoine Furetière, en 1701. Défini à ce moment-là comme un terme relevant de la médecine pour parler d'embonpoint, il ne décrit une personne malade qu'à partir de 1760. Avec le siècle des Lumières, la technologie de la santé et la volonté de maîtrise de soi propulsent le corps au centre des préoccupations mais aussi au centre des relations de pouvoir établies au travers des normes corporelles, renforcées par le discours médical.

33 Ibid., p. 59.

${ }^{34}$ K. LeBesco, « Queering Fat Bodies/Politics », in J. E. Braziel et K. LeBesco, op. cit., p. 79.

${ }^{35}$ Certaines ont eu lieu à la RainbowHouse de Bruxelles, lorsque le groupe faisait partie de l'association, d'autres ont été menées dans l'espace public, ou dans le cadre d'évènements organisés par des partenaires.

${ }^{36}$ http://www.pinkscreens.org/. Le festival est organisé annuellement par l'association Genres d'à côté dont le but est de promouvoir les sexualités et les genres différents.

37 http://www.who.int/topics/obesity/fr/. 
Dès cette période, le corps sera chiffré, mesuré et aura un rôle capital tant dans le privé que dans le social. Véritable charnière entre la place occupée par un individu dans la société et l'intimité de celui-ci, le corps sera dorénavant lu comme un reflet des responsabilités de chacun.

Si l'IMC n'est pas le seul outil de normalisation du discours médical mis en place, il est révélateur de la pensée qui s'insurge, depuis le XVIII ${ }^{\mathrm{e}}$ siècle, contre le corps gros. Dès ce moment-là, une technologie centrée sur la vie prend une importance cruciale : le corps gros est perçu comme malade (puisque la norme est la bonne santé), ce qui lui confère un statut morbide (on parlera d'ailleurs plus tard d'obésité morbide). La grosseur devient synonyme de mort, être gros est synonyme de danger.

Ce qu'il faut entendre par médicalisation est, comme le décrit Cooper $^{38}$, le processus par lequel des groupes sociaux sont définis selon des termes de leur anatomie et de leur physique afin d'être catégorisés. Ce qui sort de la normalité est considéré comme malade et, comme l'auteur l'indique, la maladie est souvent vue comme un tabou social, puisqu'elle rappelle à l'être humain son statut de mortel. Outre cet aspect, la maladie est associée à l'idée de chaos, de désordre, ce qui va à l'encontre de l'idéal de santé et d'ordre instauré depuis le siècle des Lumières. Le corps gros est donc devenu, peu à peu, victime de cette médicalisation et est dorénavant rangé dans la catégorie « corps malade », « problématique », menaçant l'ordre public. Selon l'auteure, la médicalisation est problématique en ce qu'elle définit les individus par rapport à une norme, à l'aune de laquelle ils peuvent (et doivent) être mesurés. Cette mesure (de la normalité à l'anormalité) implique une dynamique binaire entre les individus : gros/mince, blanc/de couleur, valide/invalide, etc. Considérer le discours médical comme vérité absolue tend à entériner ces catégories binaires, sans les remettre en question, et à appliquer, par conséquent, l'étiquette de « hors-normes » à tous les corps qui s'écartent de la norme, statut le plus enviable dans la société et le seul sujet possible. Chaque catégorie de corps « hors-norme » sera traitée différemment, mais relève bel et bien d'une même dynamique de pouvoir sous-tendue par le discours médical. Questionner ces catégories faussement immuables, c'est déconstruire la binarité qui rejette les corps différents dans le non-vi(v)able, et permettre aux individus de devenir sujet, d'accéder à une identité et ainsi de se réapproprier leur corps. " Décrire les personnes grosses comme obèses, c'est médicaliser la diversité des êtres humains ${ }^{39}$, et puisque le corps gros est perçu comme malade, c'est aussi pathologiser toute une partie de la population. Vouloir maintenir l'ordre public, la norme, en effaçant les corps différents, en les rejetant dans l'abject, dans l'anormal, dans le pathologique, a un prix.

Si le combat n'a pas changé, le groupe de militantisme gros belge a quelque peu évolué au fil du temps. Aujourd'hui, il n'est plus actif que sur la toile. Facebook et tumblr ${ }^{40}$ sont devenus des outils d'activisme à part entière et permettent de faire circuler informations, représentations positives, Q\&A et autres éléments cruciaux pour la propagation du mouvement. Tous les corps sont bons, certes, mais encore

${ }^{38}$ Ch. CoOper, Fat and proud, op. cit., p. 117-118.

${ }_{39}$ M. Wann, «Forword », in E. Rothblum et S. Solovay, The fat studies reader, op. cit., p. XIII, ma traduction de « calling fat people « obese » medicalizes human diversity ».

${ }^{40} \mathrm{http}: / /$ fatpositivitybelgium.tumblr.com/. 
faut-il que l'idée se répande dans les esprits. Fat Positivity Belgium prend corps virtuellement et participe activement à la communauté cyber-militante. Un outil qui n'est pas à négliger de nos jours. Cette période de transition ne ferme pas la porte à une potentielle reformation physique du groupe. A suivre, donc.

Ainsi, Fat Positivity, que ce soit à travers ses actions passées, ses collaborations ou encore ses posts sur la toile, élabore toute une stratégie militante visant à se réapproprier une identité de gros. Véritable touche-à-tout activiste, le groupe multiplie les activités et diversifie ses moyens d'actions afin de faire une place aux personnes grosses. Mettant à mal une hégémonie légitimée et bien ancrée dans les mœurs, le groupe utilise des pratiques performatives, pratiques qui dévoilent la contingence des normes corporelles véhiculées par des discours institutionnels. Il offre ainsi une visibilité nouvelle aux personnes grosses et déconstruit les discours stigmatisants. La grosseur est enfin à l'honneur.

\section{Un cas de Force Majeure}

Rien à perdre : voilà ce que nous signale la troupe australienne Force Majeure. Il n'y a plus rien à perdre, mais tout à gagner. Nothing to Lose est un spectacle de danse qui fait la part belle aux danseurs gros. Sous le feu des projecteurs, ce spectacle met à mal les conceptions hégémoniques de la grosseur et de la capacité physique des personnes grosses.

La troupe Force Majeure est un collectif d'artistes multidisciplinaire dont l'axe principal est la création de spectacles basés sur le mouvement. Actif depuis 2002, Force Majeure regroupe des danseurs, des comédiens, des écrivains, des musiciens, des réalisateurs. Avec Nothing to Lose ${ }^{41}$, présenté au festival de Sydney 2015, le collectif propose un spectacle hors-normes : des danseurs gros y dévoilent leur corps, jouent avec leur grosseur, la spectacularisent. Ce spectacle est étudié ici à partir d'images, de vidéos et de propos recueillis sur la toile, l'analysant comme un outil de sensibilisation et de représentation performative, plutôt que comme un spectacle artistique.

Sur le site internet du collectif, un mot d'explication en dit long sur l'intention du spectacle : « «Fat » is a powerful little word, full of baggage and judgement. This undaunted production delves into real-life experiences and stories to challenge aesthetic norms and reclaim a performative space for people with large bodies. (...) Nothing to Lose investigates the relentless fascination with the fat body while abandoning stereotypes and reshaping expectations $»$.

\section{Le corps comme outil de productivité}

Mais quel est l'intérêt de déconstruire les préjugés concernant la capacité physique des personnes grosses ? Allant de pair avec la médicalisation de la grosseur, le corps, depuis la révolution industrielle, se doit d'être productif. A partir du $\mathrm{XIX}^{\mathrm{e}}$ siècle $^{42}$, il est inscrit dans une démarche émancipatrice à travers le travail industriel, ce qui permet à l'individu une certaine mobilité sociale et « lui offre une vie productive, à

\footnotetext{
${ }^{41} \mathrm{https}: / /$ www.youtube.com/watch? $\mathrm{v}=52 \mathrm{nBgBEIMSY}$.

42 P. FRASER, Le corps obèse objet d'aversion, op. cit., p. 145-147.
} 
partir de laquelle il se définit socialement ${ }^{43}$. A l'ère de la révolution industrielle, le corps se fait machine et doit répondre à des critères de productivité toujours plus contraignants. Peu à peu, le corps gros est rejeté dans la catégorie improductive, socialement inacceptable. Paradoxalement, l'image du corps gros de l'homme opulent du passé n'est pas tout à fait remplacée par celle des personnes grosses issues des classes sociales défavorisées et sujettes aux moqueries ${ }^{44}$; ces deux facettes forment désormais la dualité de la stigmatisation de la grosseur actuelle. Si la grosseur reste un symbole du capitalisme, elle devient aussi signe de misère sociale, corps improductif, corps inutile.

Ainsi, la personne grosse, par défaut de gouvernance de $s o i^{45}$, ne parvient pas à trouver sa place dans la société, menace l'ordre social et, par son corps personnel, bouleverse le corps social. Voici le point de départ d'un des stigmates que porte la grosseur encore à l'heure actuelle : si l'oisiveté est la mère de tous les vices, dans une société capitaliste, le corps gros, perçu comme responsable de sa condition et donc de son improductivité, devient figure emblématique de la paresse. Mais plus encore, par son manque de volonté, il devient inapte à s'intégrer à la société et n'arrive donc pas à jouer son rôle social : son poids devient un poids pour la société. Chaque individu gros est donc pointé du doigt, problématisé, visé comme problème social à régler. Le corps individuel devient affaire sociale, le personnel devient affaire d'Etat. Voilà donc que la corpulence, la citoyenneté et la morale se voient étroitement liées, associées ${ }^{46}$ : celui qui parviendrait à se conformer aux normes démontrerait sa capacité d'autogestion des normes, et donc sa docilité. Cet individu, par sa minceur, prouverait sa productivité et donc mériterait sa position sociale privilégiée. A l'inverse, la personne grosse, par sa seule corpulence, démontrerait son inadaptabilité à la société capitaliste ainsi qu'une morale douteuse, puisqu'elle ne remplirait pas son rôle de citoyen autonome, responsable. Etre gros, dans la société capitaliste, équivaudrait à un échec ${ }^{47}$ en matière d'ascension sociale. Par conséquent, le corps gros porte deux stigmates, intrinsèquement liés : il est malade et il est improductif. Dans une société capitaliste, où la bonne santé est perçue comme un droit mais aussi (et surtout) comme une obligation, le corps gros n'aurait pas sa place.

Kate Champion, la chorégraphe du spectacle, a décidé de montrer la grosseur sur scène, après avoir constaté qu'il manquait cruellement de danseurs gros sur la scène artistique contemporaine ${ }^{48}$. Etant mince elle-même, elle a tenu à faire appel à une artiste militante grosse, Kelli Jean Drinkwater, pour mettre le spectacle sur pied. Elle explique qu'avant la création du spectacle, elle n'avait pas conscience du degré d'abus dont étaient victimes les personnes grosses et qu'elle a dû s'adapter aux

43 Ibid.

${ }^{44}$ Ibid., p. 158-159.

${ }^{45}$ Fraser ajoute que ce défaut de gouvernance de soi provient de trois axes principaux : le devoir d'équilibre, le devoir d'attention et d'effort, et le devoir de maitrise et de restriction. Ce manque de gouvernance de soi va à l'encontre de l'idéal d'autonomie individuelle, héritage du siècle des Lumières (Ibid., p. 141).

${ }^{46}$ K. LeBesco, Revolting bodies ?, op. cit., p. 55.

47 Ibid.

$48 \mathrm{https}: / /$ www.youtube.com/watch?time_continue=18\&v=7N5DpIairL8. 
spécificités physiques des danseurs, qui, certes, ne pouvaient peut-être pas accomplir certains pas, mais étaient capables de faire bouger leur chair et leur corps comme une personne mince ne le pourrait jamais. Par ailleurs, si Kate Champion ne se revendique pas activiste, elle est toutefois consciente de l'aspect politique d'un tel spectacle et précise que c'est son rôle en tant qu'artiste de faire bouger les choses dans la société. Il est crucial d'avoir cette complémentarité ; d'une part, des groupes de militantisme gros et d'autre part, des artistes qui mettent à mal les conceptions hégémoniques de la grosseur.

La stratégie performative dans Nothing to Lose est éminemment identitaire : rendre visible un corps effacé de l'espace public, le montrer en train de déconstruire les préjugés liés à la grosseur et à sa soi-disant incapacité physique, c'est justement en finir avec la liminalité de l'identité de gros. Mais plus encore, si l'on en croit l'adage " you can't be what you can't see », il parait évident qu'une représentation positive de la grosseur est nécessaire pour accéder à une identité de gros non liminale.

\section{Le corps comme cour du processus identitaire}

Le corps est un terrain de significations, traversé par des discours normalisateurs, sans cesse présenté, représenté et performé ${ }^{49}$ et se trouve au cœur du processus identitaire. Le corps, quel qu'il soit, est le lieu privilégié de signification : il représente un carrefour de significations entre le personnel et le social ${ }^{50}$. Afin d'avoir un statut social, une identité, il est nécessaire de se conformer à la norme, sous peine de se voir exclu, marginalisé. Ainsi, le corps a pour visée de rendre visible notre capacité personnelle à nous conformer aux attentes de la société régulatrice ${ }^{51}$.

En effet, la norme est productrice : elle « implique une logique de sujétion, une forme-sujet, qui se constitue dans cela même qui l'assujettit ${ }^{52}$. En d'autres termes, pour devenir sujet (ou avoir une identité sociale), il est nécessaire de se conformer à la norme. Cette soumission est volontaire et n'est pas ressentie comme contraignante, et ainsi le sujet se forme selon un " étalon de normalité ${ }^{53}$. Mais le corps gros est subversif : il ne correspond pas à la norme, et lorsqu'il n'accepte pas son statut « horsnorme », il devient liminal. La grosseur qui s'assume et se revendique est radicale. Une fois présenté, représenté et performé, le corps gros devient gros.

\section{Conclusion}

Le corps est la matière première des performances culturelles, ancrées dans un contexte culturel donné ${ }^{54}$ et véritable carrefour de relations de pouvoir entre individus, véhiculées notamment à travers les normes. Grâce au concept de liminalité

${ }^{49} \mathrm{Ph}$. VAnNini et D. Waskuls (éd.), Body/embodiment : Symbolic interaction and the sociology of the body, Ashgate Publishing, 2012 p. 3.

${ }^{50}$ Ibid.

${ }^{51}$ Ibid., p. 70.

${ }^{52}$ F. BRUGÈRE et G. Le BlanC (éd.), Judith Butler : trouble dans le sujet, trouble dans les normes, Paris, PUF, 2009 p. 10.

${ }^{53}$ Ibid., p. 11.

${ }^{54}$ J. L. LewIs, The Anthropology of Cultural Performance, New York, Palgrave Macmillan, 2013. 
et de performance, nous constatons que les artistes et militants gros mettent à mal les relations de pouvoir véhiculées par les normes corporelles, déconstruisent les discours hégémoniques sur la grosseur, mettent en place des pratiques performatives qui s'inscrivent dans des stratégies militantes visant à promouvoir la diversité et l'acceptation de soi et à en finir avec les diktats de beauté et de santé ancrés dans une société patriarcale et capitaliste. Fat Positivity offre un espace de revendications, espace physique et virtuel, où le corps gros est célébré - mais pas seulement : en effet, le groupe tient à son intersectionnalité et prône une diversité corporelle radicale. « Tous les corps sont bons ! » La troupe Force Majeure, quant à elle, propose un spectacle tout à fait subversif car dansé et décomplexé. Spectacularisant la grosseur, dévoilant et jouant avec la chair, le spectacle confronte les spectateurs à leurs conceptions hégémoniques de ce qu'est un corps de danseur et donc de ce qu'il faut entendre par la « bonne santé ».

En conclusion, pour permettre aux personnes grosses d'exister en tant que sujet, pour créer une identité de gros qui ne dépende pas des stéréotypes engendrés par le modèle hégémonique, pour envisager une subversion de la norme corporelle, peutêtre faudrait-il considérer l'identité de gros, non plus comme liminale, mais bien comme permanente. Si l'on associe ce changement à une déconstruction du discours hégémonique sur la grosseur, par une voie corporelle, alors peut-être arriveronsnous à resignifier l'identité grosse. Loin des carcans hégémoniques, les artistes et militants proposent une autre vision de la grosseur, une autre identité de gros, une autre représentation des personnes grosses et en finissent avec la liminalité de leur identité : ils sont présents, sans complexe, et résolument gros. Les discours alternatifs sur la grosseur, les revendications, la représentation positive des personnes grosses sont autant d'éléments nécessaires pour démanteler l'hégémonie de la minceur, la liminalité de l'identité de gros et briser le tabou autour du « gros mot » qu'est devenu le mot « gros ». 